\title{
Combined impact of extended criteria donor and cold ischemic time on delayed graft function in deceased donor kidney transplantation
}

\author{
Seung Hwan Song, Dami Jung, Ku Yong Chung
}

Department of Surgery-Transplantation, Ewha Womans University Seoul Hospital, Seoul, Korea

Background: The most significant complication is delayed graft function (DGF) on deceased donor kidney transplantation (DDKT). Multiple factors belonging to donor, recipient, and transplant procedures have an effect on the development of DGF. We aim to evaluate the combined impact of extended criteria donor (ECD) and cold ischemic time (CIT) of DGF and its effects on the graft. Methods: Between January 2008 and October 2020, a total 99 recipients who underwent DDKT were retrospectively reviewed. We classified recipients into two groups: $\operatorname{DGF}(-)$ vs. DGF(+). Each group was subdivided according to Korea Network for Organ Sharing (KONOS) ECD criteria. The risk factors of DGF associated with donor and recipient were analyzed. The effects of DGF were examined on the graft function and survival.

Results: We included 99 DDKT cases. Among 99 DDKT, 35 cases were included in DGF $(+)$, and the others were in DGF(-) $(n=64)$. The serum creatinine level before donor nephrectomy of the DGF $(+)$ group was significantly higher $(1.7 \pm 1.1 \mathrm{mg} / \mathrm{dL})$ than that of the DGF $(-)$ group $(1.0 \pm 0.5 \mathrm{mg} / \mathrm{dL}, \mathrm{P}<0.0001)$. CIT of the $\mathrm{DGF}(+)$ group was $333.2 \pm 95.5$ minutes, compared to $289.4 \pm 62.3$ minutes of the DGF $(-)$ group $(\mathrm{P}=0.018)$. In the DGF $(+)$ group, the ECD group showed shorter CIT than SCD groups statistically $(301.2 \pm 92.5$ minutes vs. (371.1 \pm 87.0 minutes, $P=0.029)$. There was no significant difference in graft function and survival between $D G F(+)$ and DGF(-) groups.

Conclusions: In this study, graft function and survival after DGF(+) DDKT were similar to that of DGF(-) DDKT. However, to reduce DGF, efforts are needed to reduce CIT in DDKT using ECD than when underwent DDKT using SCD. 